\title{
Whole body vibration training as the novel anti- aging trend in medicine and gerontology and its impact on the immune status of an elderly person
}

\begin{abstract}
The neuromuscular stimulation by Whole Body Vibration (WBV) has found an increasing common application in restorative medicine and gerontology. Despite numerous effects of WBV as a training method on performance and its ability to aid in the rehabilitation of chronic diseases, musculoskeletal and neurological disorders, in the review of the current literature there is no sufficient data indicating its impact on the immune system, especially in elderly. Authors studied «immunological safety» of such training in its long-term program. The results of 24 -week training proved that $\mathrm{WBV}$ is an objectively benign type of intervention.
\end{abstract}

Keywords: whole body vibration, aging, immune system, activation antigens, antiaging medicine, rehabilitation
Volume 3 Issue I - 2018

\author{
Shirolapov IV, Pyatin VF, Nikitin OL \\ Department of Physiology, Samara State Medical University, \\ Russia
}

Correspondence: Igor Shirolapov, Department of Physiology Samara State Medical University, 89 Chapaevskaya str, Samara, Russia,Email ishirolapov@mail.ru

Received: November 28, 2017 | Published: January 25, 2018

\section{Introduction}

Aging is a programmed physiological process, while the aging rate can be different, and can even be controlled. Currently, antiaging medicine solves the issues of increasing the duration, quality of life and slowing down the internal aging processes. Recently, along with the traditional physical exercises in people of different ages, the physiological effects of Whole Body Vibration (WBV) training are applied (acute exercises and long-term programs of neuromuscular rehabilitation). Such innovative physical influence leads to intensive neurogenic adaptation, which as a result finds application not only in sports, but also in restorative medicine and gerontology. ${ }^{1-9}$

For each physiological state, including physiological aging, there is a certain level of stress, when it exceeds a general disturbance of metabolism and immune response. Since the character of the immune response is interrelated with the activity of immunocompetent cells, the analysis of the activation profile of lymphocytes is the promising direction of immunological studies. Increase in the expression of activation antigens may coincide with an exacerbation of the pathological process, correlates with immune activity or expresses its ability and "willingness" to respond to environmental factors, including various pathogens and stressors.

The neuromuscular stimulation by WBV has found an increasing application in the fields of professional sports and medicine, but the effect of such influence on the immune system remains poorly understood. In the review of the literature there is no sufficient data indicating the effect of the WBV on the immune system especially in elderly. ${ }^{10,11}$ So the authors in their practice decided to perform a clinical study of the «immunological safety» of such training (especially in elderly patients) in its long-term program.

\section{Methods and technique}

The number of cells expressing of CD markers was studied by flow cytometer «Facs Calibur» (Becton Dickinson, USA) in 39 women (WBV \& control groups, $67 \pm 4$ yrs, 19 and 20 participants in experimental and control group, respectively). The contents of the following subpopulations of lymphocytes was studied: $\mathrm{CD}^{+}$,
$\mathrm{CD}^{+}{ }^{+} \mathrm{CD} 4^{+}, \mathrm{CD}^{+}{ }^{+} \mathrm{CD} 8^{+}, \mathrm{CD} 3{ }^{-} \mathrm{CD} 8^{+}, \mathrm{CD} 3{ }^{-} \mathrm{CD} 16^{+}, \mathrm{CD} 3^{+} \mathrm{CD} 16^{+}, \mathrm{CD} 3$ $\mathrm{CD} 20^{+}, \mathrm{CD} 25^{+}, \mathrm{CD}^{+}{ }^{+} \mathrm{CD} 25^{+}, \mathrm{CD}^{+}{ }^{+} \mathrm{DR}^{+}$cells. The authors also studied serum concentrations of immunoglobulins $\mathrm{A}, \mathrm{M}, \mathrm{G}$ and pro-inflammatory cytokines TNF $\alpha$ and IL- 8 . The patients from the control group were not involved in the program of WBV training. Additionally noted that patients from both study groups received no additional physical exertion and followed the same diet.

Neuromuscular stimulation used the «Power Plate $\left.{ }^{\mathbb{B}}\right\rangle$. Rehabilitation mode: load on the principle of progression 3 times/week for $30 \mathrm{~min}$, frequency $30-40 \mathrm{~Hz}$ and amplitude is low, duration 24 weeks.

\section{Results and discussion}

The level of CD25+ expression (as the marker of the early stage of activation) remained practically unchanged throughout the study program in both groups. Nevertheless, in WBV-group the absolute number of CD25+ cells decreased slightly by the 24th week of program. The absolute number of T-lymphocytes expressing the late activation marker (HLA-DR+) in WBV-group was the lowest in 12 weeks of the study ( $40 \%$ decrease, $p<0.05$ ). In the control group there was a gradual decrease in the absolute also.

Simultaneously, the following changes in the immunological profile of the subjects were noted. In the rehabilitation WBV group, the decrease in $\mathrm{CD}^{-} \mathrm{CD} 16^{+}$lymphocyte count after 12 weeks was $14 \%$, and after 24 weeks - $13 \%$ compared to the initial value $(\mathrm{p}<0.05)$. The relative level of $\mathrm{CD}^{+}$cells in women in the WBV group was maximal after 12 weeks of the study $(\mathrm{p}<0.05)$, and after 12 weeks of the experiment there was a slight decrease in the absolute and relative $\mathrm{CD}^{+} \mathrm{DR}^{+}$cell count in women from this group. The levels of pro-inflammatory cytokines in the experimental WBV group increased after 12 weeks (IL- 8 level increased by $42 \%$, serum TNF $\alpha$ level increased by $101 \%$, compared at the start of the study, $\mathrm{p}<0.05$ ). The authors found no significant changes in Ig A, G, M during the 24 weeks of WBV training. At the end-point of the research, the studied immune parameters returned to the initial values, despite the progressive increase in the WBV training load in the period from 12 to 24 weeks of the experiment. 
The authors suggest the mechanisms of the observed changes involved mechanoreceptor activation of muscle contractions, production of hormones, stimulation of muscle production of IL-6 and pro-inflammatory cytokines, in particular IL- 8 and TNF $\alpha$.

At present, the problems of anti-aging physiology in the fields of geriatrics and gerontology induce increasing scientific basic and clinical interest. Anti-aging direction in medicine includes aspects of the physiology of aging, medical and functional treatment of emerging age-related changes in physiological functions and the search for antiaging strategies in the way of life of a person.

Investigation of the impact of WBV training, including longterm neuromuscular stimulation, which causes acute and prolonged physiological effects in the human and animal organism, is actualized by the perspective use of modern innovative technologies of medical rehabilitation. ${ }^{1,12}$ There is a significant clinical efficacy in the treatment and prevention of osteoporosis, muscle hypotrophy and postural control in elderly, fibromyalgia, disorders of movement (in patients after stroke or with Parkinson's disease and multiple sclerosis)..$^{2-9}$

It is known that functionally active, "working" muscles are considered as an immunoendocrine organ on the basis that skeletal muscles synthesize and secrete a large number of regulatory peptidemyokines and cytokines, in particular IL-6. ${ }^{13}$ Physical load as a stress factor has a dose-dependent effect on the immune function: it does not cause pathological changes up to certain levels; moderate physical activity can have a stimulating effect on immunity indices; however, heavy physical loads, the level of which is not comparable with the current physiological state of the organism, may cause an immunosuppressive effect. ${ }^{14}$

At the same time, it should be noted that the character of the immune response is directly related to the activity of various immunocompetent cells. Thus, exacerbation of atopic diseases is associated with intensive activation processes in the immune system, which are accompanied by a marked increase in the expression of early (in particular, CD25, CD71) activation antigens. Expression of the same late (HLA-DR) antigens is directly related to the severity of the disease. Also in professional athletes during intensive training cycles, there is a significant decrease in the relative content of CD25+ cells and serum immunoglobulins, which indicates a decrease in the functional resources of T- and B-lymphocytes and may cause a depression in immune resistance to infections as a consequence. ${ }^{15} \mathrm{~A}$ comparative analysis of activation processes in the immune system in current study showed that the levels of expression of activation markers remained practically unchanged after the completion of the WBV program, and therefore the reserve capacities of lymphocytes persist at a high functional level and do not induce further the appearance of immunosuppression.

\section{Conclusion}

The results indicate the changes in the immunological indices in elderly patients after 12 weeks of Whole Body Vibration program. At this time, the highest level of physical load was noted according to the principle of progression. After 24 weeks of WBV training, the serum levels of pro-inflammatory cytokines, immunoglobulins and content of the immune cells did not differ significantly from the baseline values. At the same time, we did not notice any clinical signs of worsening of patient's self-feelings. On this basis, it can be concluded that WBV training during non-depleting mode of loading does not refer to strong stress factors for the function of the immune system, in particular for the elderly. In our study, it was shown that longterm neuromuscular stimulation by WBV is therefore an objectively safe type of rehabilitation and can be used in the fields of restorative medicine and gerontology.

\section{Acknowledgments}

None.

\section{Conflict of interest}

Authors declare that there are no conflicts of interest.

\section{References}

1. Chanou K, Gerodimos V, Karatrantou K, et al. Whole-body vibration and rehabilitation of chronic diseases: a review of the literature. J Sports Sci Med. 2012;11(2):187-200.

2. Dionello CF, Sá-Caputo D, Pereira HV, et al. Effects of whole body vibration exercises on bone mineral density of women with postmenopausal osteoporosis without medications: novel findings and literature review. $J$ Musculoskelet Neuronal Interact. 2016;16(3):193-203.

3. Haas CT, Turbanski S, Kessler K, et al. The effects of random whole-body-vibration on motor symptoms in Parkinson's disease. NeuroRehabilitation. 2006;21(1):29-36.

4. Kotel'nikov GP, Piatin VF, Bulgakova SV, et al. Whole body vibration (acceleration) training increases bone mineral density and serum levels of osteocalcin in elderly women. Adv Gerontol. 2010;23(2):257-262.

5. Piatin VF, Shirolapov IV, Nikitin OL. Vibration physical exercises as the rehabilitation in gerontology. Adv Gerontol. 2009;22(2):337-342.

6. Rogan S, Taeymans J, Radlinger L, et al. Effects of whole-body vibration on postural control in elderly. BMC Geriatrics. 2011;73:95-112.

7. Torvinen S, Kannus P, Sievänen H, et al. Effect of 8-month vertical whole body vibration on bone, muscle performance and body balance: a randomized controlled study. J Bone Miner Res. 2003;18(5):876-884.

8. Turner S, Torode M, Climstein M, et al. A randomized controlled trial of whole body vibration exposure on markers of bone turnover in postmenopausal women. J Osteoporos. 2011;710387.

9. Van der MG, Zeinstra E, Tempelaars J, et al. Handbook of Acceleration Training. CA: Healthy Leaning, USA; 2007. p. 181

10. Campbell PT1, Wener MH, Sorensen B, et al. Effect of exercise on in vitro immune function: a 12-month randomized, controlled trial among postmenopausal women. J Appl Physiol. 2008;104(6):1648-1655.

11. Ginaldi L, De Martinis M, D’Ostilio A, et al. The immune system in the elderly: I. Specific humoral immunity. II. Specific cellular immunity. III. Innate immunity. Immunol Res. 1999;20(2):101-117.

12. Pyatin VF, Kolsanov AV, Shirolapov IV. Recent Medical Techniques for Peripheral Nerve Repair: Clinico-Physiological Advantages of Artificial Nerve Guidance Conduits. Adv Gerontol. 2017;7(2):148-154.

13. Iizuka K, Machida T, Hirafuji M. Skeletal muscle is an endocrine organ. J Pharmacol Sci. 2014;125(2):125-131.

14. Pedersen BK, Hoffman GL. Exercise and the immune system: regulation, integration, and adaptation. Physiol Rev. 2000;80(3):1055-1081.

15. Gleeson M. Immune function in sport and exercise. J Appl Physiol. 2007;103(2):693-699. 